Research Article

\title{
A prospective study to analyse antibiotic susceptibility pattern of Pseudomonas aeruginosa in a tertiary care hospital
}

\author{
Syed S. Ameen ${ }^{1}$, Laxminarayana Bairy K. ${ }^{2}$, Mohammed Imran ${ }^{3}$, Indira Bairy ${ }^{4}$
}

\begin{abstract}
${ }^{1}$ Department Pharmacology, Dr B.R. Ambedkar Medical College, Bangalore, India, ${ }^{2}$ Department Pharmacology, Kasturba Medical College, Manipal, India

${ }^{3}$ Department of Community Medicine, Dr BR Ambedkar Medical College, Bangalore, India

${ }^{4}$ Department of Microbiology, Manipal University, India
\end{abstract}

Received: 01 June 2016

Accepted: 06 June 2016

*Correspondence to:

Dr. Syed S. Ameen,

Email: ameensalaam85

@ gmail.com

Copyright: (c) the author(s), publisher and licensee Medip Academy. This is an openaccess article distributed under the terms of the Creative Commons Attribution NonCommercial License, which permits unrestricted noncommercial use, distribution, and reproduction in any medium, provided the original work is properly cited.

\begin{abstract}
Background: Pseudomonas aeruginosa, a gram-negative pathogen is commonly associated with nosocomial infections. Infections caused by $P$. aeruginosa can range from superficial skin infections to fulminant sepsis. Antimicrobial resistance is an area of prime concern in pseudomonal infections. The objective of the study was to evaluate and analyse the antibiotic susceptibility pattern of $P$. aeruginosa at a tertiary care hospital in South India.

Methods: The study was carried out at Kasturba Medical College and Hospital, Manipal, India from January 2011 to December 2011. Ten different types of specimens were collected from patients who were culture positive for Pseudomonas aeruginosa. Antibiotic susceptibility was confirmed by disk diffusion technique on Muller-Hinton medium and was performed according to the Clinical Laboratory Standard Institute (CLSI) guidelines.

Results: Out of 200 samples of $P$. aeruginosa $69.5 \%$ and $30.5 \%$ were from male and female patients respectively. Majority of the specimen from which $P$. aeruginosa was isolated consisted of sputum, pus and urine. Among the antimicrobial drugs tested, organism was most sensitive to carbapenems (77.5\%), piperacillin-tazobactam (77\%) and cefoperazone-sulbactam $(72 \%)$. Resistance rates were high for fluoroquinolones (FQs) (43.5\%), gentamicin $(40.5 \%)$, tobramycin $(40.5 \%)$, ticarcillin-clavulanic acid $(39 \%)$ and aztreonam $(38 \%)$ when compared to cefepime $(31.5 \%)$, ceftazidime $(32.5 \%)$, netilmicin $(34.5 \%)$ and amikacin $(35 \%)$.

Conclusions: Carbapenems and piperacillin-tazobactam were the best antipseudomonal agents with highest sensitivity to $P$. aeruginosa. FQs, gentamicin and tobramycin were the least effective drugs against $P$. aeruginosa as monotherapy.
\end{abstract}

Keywords: $P$. aeruginosa, Antibiotic susceptibility, Antipseudomonal agents, Nosocomial infections

\section{INTRODUCTION}

Pseudomonas aeruginosa, a gram-negative pathogen is commonly associated with nosocomial infections. It is involved in wide variety of human infections, ranging from superficial skin infections, acute and chronic lung infections to fulminant sepsis. It is distinguished as an opportunistic pathogen causing infection in patients with defective physical, phagocytic and immunological defense mechanisms. ${ }^{1}$
Historically, it was considered a major burn wound pathogen, an agent of bacteremia in neutropenics and the most common pathogen in cystic fibrosis patients. However, these interesting associations have undergone considerable changes. Now $P$. aeruginosa is the $2^{\text {nd }}$ most common cause of nosocomial pneumonia $(17 \%), 3^{\text {rd }}$ important etiology for urinary tract infection $(7 \%), 4^{\text {th }}$ most common cause of surgical site infection, $5^{\text {th }}$ most common isolate $(9 \%)$ overall from all sites and $7^{\text {th }}$ most frequently isolated pathogen from the bloodstream. ${ }^{2}$ 
Antimicrobial resistance (AMR) to broad spectrum antibiotics is an area of prime concern in pseudomonal infections. ${ }^{1,3}$ It is the most widespread multidrug-resistant (MDR) gram-negative pathogen causing pneumonia in hospitalized patients. It not only limits therapeutic options but also affects clinical outcome by increasing morbidity and mortality. ${ }^{4}$ Therefore, a study was conducted to determine the current antibiotic sensitivity and resistance rates which would help in laying down current recommendations for empirical antibiotic regimens for treating $P$. aeruginosa infections and also to minimize progression of MDR.

The objective of the study was to analyse antibiotic susceptibility patterns of $P$. aeruginosa in study population.

\section{METHODS}

The study was conducted in Kasturba Hospital, Manipal, Karnataka, India over a period of one year (January to December 2011). Study subjects included were patients aged above 18 years of either sex who got admitted to the hospital during study period with positive growth for $P$. aeruginosa. Subjects were explained about the study and written informed consent was obtained. Cases from burns ward and OPD were excluded from study.

A total of 200 patients with positive culture for $P$. aeruginosa were included in the study after considering inclusion and exclusion criteria. Ethical clearance was obtained from institutional ethical committee before the start of study.

Pre-designed proforma was used to collect demographic details, laboratory data and treatment information of the patient. Microbiological data including specimen, site, presence of any associated organism, antibacterial sensitivity and resistance patterns of $P$. aeruginosa was noted.

\section{Microbiology}

Culture examination was carried out using blood agar, nutrient agar and MacConkey's agar, followed by study of colony morphology, pigment production, positive oxidase reaction and oxidase in oxidation fermentation medium. ${ }^{5}$ Antibiotic susceptibility was confirmed by disk diffusion technique on Muller-Hinton medium and was performed according to the Clinical Laboratory Standard Institute (CLSI) guidelines. Paper disks were impregnated with antibiotics. These were commercially procured from span diagnostics.

Antibiotics which were tested for sensitivity are ceftazidime, cefoperazone-sulbactam, cefepime or cefpirome, piperacillin, piperacillin-tazobactam, ticarcillin-clavulanic acid, aztreonam, carbapenem, gentamicin, tobramycin, amikacin, netilmicin and ciprofloxacin or levofloxacin.
Cultures were incubated overnight at $37^{\circ} \mathrm{C}$. Diameter of the zone of inhibition was measured and compared to that of standard strain and results were interpreted as sensitive and resistant, based on CLSI guidelines. ${ }^{6}$

\section{Statistical analysis}

Analysis was primarily descriptive. Data thus obtained was entered in excel sheet. Analysis was done using SPSS version 17.0 as percentages and proportions.

\section{RESULTS}

Out of 200 cases analysed in our study, there were $141(70.5 \%)$ males and $59(29.5 \%)$ females and majority of them were aged 40 and above (Table 1). Various specimens were studied including pus-wound swab, sputum, urine, blood, etc. $P$. aeruginosa was most commonly isolated from sputum $(31.5 \%)$ followed by pus-wound swab $(25.5 \%)$ and urine $(22.5 \%)$ (Table 2$)$.

Table 1: Age and sex distribution of study subjects.

\begin{tabular}{|llll|}
\hline $\begin{array}{l}\text { Age group } \\
\text { (years) }\end{array}$ & Males (\%) & Females (\%) & Total (\%) \\
\hline $18-40$ & 23 & 21 & 44 \\
\hline $41-60$ & 57 & 22 & 79 \\
\hline$>60$ & 59 & 18 & 76 \\
\hline Total & 139 & 61 & 200 \\
\hline
\end{tabular}

Table 2: Microbiological specimens with positive culture for $\boldsymbol{P}$. aeruginosa.

\begin{tabular}{|lll|}
\hline Specimen & $\begin{array}{l}\text { Total no. of } \\
\text { cases }\end{array}$ & $\begin{array}{l}\text { Percentage } \\
\text { of cases }\end{array}$ \\
\hline Pus and wound swab & 51 & $25.5 \%$ \\
\hline Sputum & 63 & $31.5 \%$ \\
\hline Urine & 45 & $22.5 \%$ \\
\hline Blood & 25 & $12.5 \%$ \\
\hline Others* & 16 & $8.0 \%$ \\
\hline
\end{tabular}

*ear swab, tracheal swab, pleural fluid, endotracheal aspirate, bronchoalveolar lavage and intercostal drainage

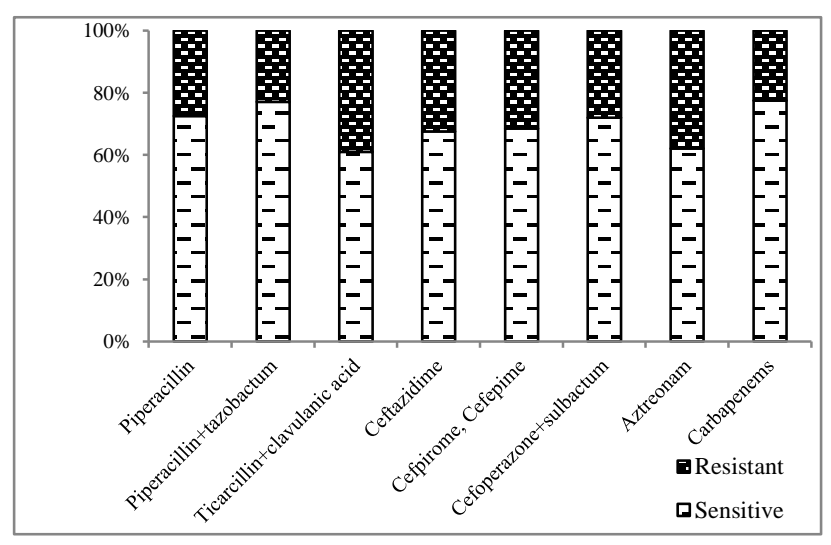

Figure 1: In vitro susceptibilities of $P$. aeruginosa to anti-pseudomonal $\beta$-lactam antibiotics. 
Susceptibility to anti-pseudomonal $\beta$-lactam antibiotics in decreasing order of frequency was as follows: carbapenems $(77.5 \%)$, piperacillin-tazobactam $(77 \%)$, piperacillin $(72.5 \%)$, cefoperazone-sulbactam $(72 \%)$, cefpirome or cefepime $(68.5 \%)$, ceftazidime $(67.5 \%)$, aztreonam (62\%) and ticarcillin-clavulanic acid (61\%) (Figure 1).

Percentage of susceptible isolates for aminoglycoside group of antibiotics was $65.5 \%$ (netilmicin), $65 \%$ (amikacin), $59.5 \%$ (tobramycin) and $59.5 \%$ (gentamicin). The fluoroquinolones (FQs) tested were ciprofloxacin and levofloxacin, $56.5 \%$ of isolates were susceptible to FQs (Figure 2).

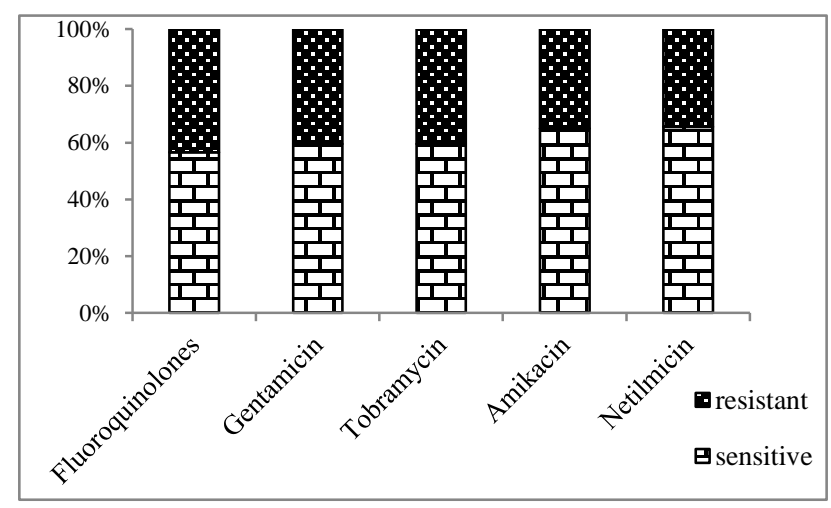

Figure 2: In vitro susceptibilities of $P$. aeruginosa to fluoroquinolones and aminoglycosides.

\section{DISCUSSION}

Out of 200 cases analysed in our study $P$. aeruginosa infection was more in patients with age above 40 years with a male preponderance $(69.5 \%)$, while females constituted only $30.5 \%$ of cases. This was consistent with a similar study done in India. ${ }^{7}$ Highest number of $P$. aeruginosa was isolated from sputum making the organism the most common cause of respiratory tract infection amongst all hospital acquired infections (HAIs). It is amongst the most common causes of ventilator associated pneumonia and carries the highest mortality among HAIs. It also harbours in damaged bronchi of bronchiectasis patients leading to acute exacerbations. ${ }^{4}$

Among the $\beta$-lactam antibiotics tested, carbapenems and piperacillin-tazobactam showed least resistance rates. These antibiotics have broader spectrum of activity than most $\beta$-lactam antibiotics, therefore were most effective against treating $P$. aeruginosa infections. Concurrent administration of $\beta$-lactamase inhibitor tazobactam enhanced antipseudomonal sensitivity of an extended spectrum penicillin like piperacillin when compared to piperacillin alone. Sensitivity rates of cefepime and cefpirome were slightly superior to ceftazidime due to their non-susceptibility to inducible $\beta$-lactamases. Cefoperazone-sulbactam was superior among all cephalosporins.
We observed that aminoglycosides and FQs were less effective when compared to most of $\beta$-lactam antibiotics. Netilmicin and amikacin were most effective among the aminoglycosides. Among all the drugs tested FQs showed highest resistance rates followed by gentamicin and tobramycin, hence these drugs should be avoided as monotherapy especially in MDR P. aeruginosa infections.

Multidrug resistance phenotypes are slowly increasing in prevalence among $P$. aeruginosa isolates. ${ }^{8-11}$ However, comparison between studies is often difficult, because definitions of multidrug resistance have not been uniform. ${ }^{9,10,12}$ The reasons for increasing nosocomial spread of MDR isolates may include lack of adherence to approved infection control policies in hospitals, increasing or cumulative antimicrobial use, and changes in the public health infrastructure. ${ }^{12-16}$

In conclusion, susceptibility of antipseudomonal agents against different isolates has decreased. Significant reduction in susceptibilities of $P$. aeruginosa isolates may compromise the ability to choose efficacious empirical regimens for treatment of this formidable pathogen especially in critically ill patients. The present study provides valuable information related to emerging trends in antimicrobial resistance to monotherapy, which is vital for clinicians in the selection of reliable empirical regimen for treating $P$. aeruginosa infections.

\section{ACKNOWLEDGEMENTS}

Author would like acknowledge Dr. Muralidhar Varma, Associate Professor, Department of Medicine, Kasturba Medical College, Manipal, for guiding and encouraging me throughout my study. I also thank Dr. Amrutha Tripathi, Dr. Shahabuddin Soherwardi and Dr. Asfiya Anjum for their timely help during data collection and analysis. Last but not the least author would like to thank Dr. Shanmukananda P, Professor and Head, department of pharmacology, Dr. B R Ambedkar Medical College, Bangalore for his advice and support.

Funding: No funding sources

Conflict of interest: None declared

Ethical approval: The study was approved by the Institutional Ethics Committee

\section{REFERENCES}

1. Kanj SS, Sexton DJ. Epidemiology and pathogenesis of Pseudomonas aeruginosa infection. Available at http: // www.uptodate.com/ contents/ epidemiologyandpathogenesis-of-pseudomonas aerug inosa infection?source=search_result \& search = pseudomonas + aeruginosa \& selectedTitle $=3 \sim 150$. Accessed on 4 March 2016.

2. National nosocomial infection surveillance (NNIS) system report: data summary from January 1992 through June 2003. Available at: 
http://www.cdc.gov/ncidod/dhqp/pdf. Accessed on 4 March 2016.

3. Ramphal R. Infections due to Pseudomonas species and related organisms. In: Longo DL, Fauci AS, Kasper DL, Hauser SL, Jamson JL, Loscalzo J, editors. Harrison's Principles of Internal Medicine. $18^{\text {th }}$ edition. New York: McGraw-Hill Companies; 2012:1266-1273.

4. Pier GB, Ramphal R. Pseudomonas aeruginosa. In: Mandell GL, Bennett JE, Dolin R, editors. Mandell, Douglas and Bennett's Principles and Practice of Infectious Diseases. $7^{\text {th }}$ edition. New York: Churchill Livingstone Elsevier; 2010:2835-2857.

5. Genner S, Ak O, Benzonana N, Batirel A, Ozer S. Susceptibility pattern and cross-resistance of antibiotics against Pseudomonas aeruginosa in a teaching hospital of Turkey. Ann Clin Microbiol Antimicrob. 2002;1:2-7.

6. National committee for clinical laboratory standards. Performance standards for antimicrobial disk susceptibility tests. Approved standard M2 A7 NCCLS. Villanova;1995:15.

7. Javiya VA, Ghatak SB, Patel KR, Patel JA. Antibiotic susceptibility patterns of Pseudomonas aeruginosa at a tertiary care hospital in Gujarat, India. Indian J Pharmacol. 2008;40(5):230-4.

8. Bert F, Maubec E, Bruneau B, Berry P, Lambert ZN. Multiresistant Pseudomonas aeruginosa outbreak associated with contaminated tap water in a neurosurgery intensive care unit. J Hosp Infect. 1998;39(1):53-62.

9. Chen HY, Yuan M, Ibrahim EIB, Livermore DM. National survey of susceptibility to antimicrobials amongst clinical isolates of Pseudomonas aeruginosa. J Antimicrob Chemother. 1995;35(4):521-34.

10. Fass RJ, Barnishan J, Solomon MC, Ayers LW. Invitro activities of quinolones, b-lactams, tobramycin, and trimethoprim-sulfamethoxazole against nonfermentative gram-negative bacilli. Antimicrob Agents Chemother. 1996;40(6):1412-8.

11. Sofianou D, Tsakris A, Skoura L, Douboyas J. Extended high-level cross-resistance to antipseudomonal antibiotics amongst Pseudomonas aeruginosa isolates in a university hospital. J Antimicrob Chemother. 1997;40(6):740-2.

12. Gales AC, Jones RN, Turnidge J, Rennie R, Ramphal R. Characterization of Pseudomonas aeruginosa isolates: occurrence rates, antimicrobial susceptibility patterns, and molecular typing in the global sentry antimicrobial surveillance program, 1997-1999. Clin Infect Dis. 2001;32(2):146-55.

13. Jones RN, Kirby JT, Beach ML, Biedenbach DJ, Pfaller MA. Geographic variations in activity of broad-spectrum b-lactams against Pseudomonas aeruginosa: summary of the worldwide sentry antimicrobial surveillance program (1997-2000). Diagn Microbiol Infect Dis. 2002;43:239-43.

14. Landman D, Quale JM, Mayorga D, Adedeji A, Vangala K, Ravishankar J, et al. Citywide clonal outbreak of multi resistant Acinetobacter baumannii and Pseudomonas aeruginosa in Brooklyn, NY: the preantibiotic era has returned. Arch Intern Med. 2002;162(13):1515-20.

15. Safdar N, Maki DG. The commonality of risk factors for nosocomial colonization and infection with antimicrobial-resistant Staphylococcus aureus, enterococcus, gram-negative bacilli, clostridium difficile, and candida. Ann Intern Med. 2002;136(11):834-44.

16. Wenzel RP, Wong MT. Managing antibiotic useimpact of infection control. Clin Infect Dis. 1999;28(5):1126-7.

Cite this article as: Ameen SS, Bairy LK, Imran M, Bairy I. A prospective study to analyse antibiotic susceptibility pattern of Pseudomonas aeruginosa in a tertiary care hospital. Int J Basic Clin Pharmacol 2016;5:1311-4. 